\title{
Inflammation in subcutaneous adipose tissue: relationship to adipose cell size
}

\author{
T. McLaughlin • A. Deng • G. Yee • C. Lamendola • \\ G. Reaven • P. S. Tsao $\cdot$ S. W. Cushman • A. Sherman
}

Received: 14 July 2009 / Accepted: 16 July 2009 /Published online: 9 October 2009

(C) Springer-Verlag 2009

\begin{abstract}
Aims/hypothesis Inflammation is associated with increased body mass and purportedly with increased size of adipose cells. We sought to determine whether increased size of adipose cells is associated with localised inflammation in weight-stable, moderately obese humans.

Methods We recruited 49 healthy, moderately obese individuals for quantification of insulin resistance (modified insulin suppression test) and subcutaneous abdominal adipose tissue biopsy. Cell size distribution was analysed with a multisizer device and inflammatory gene expression with real-time PCR. Correlations between inflammatory gene expression and cell size variables, with adjustment for sex and insulin resistance, were calculated.

Results Adipose cells were bimodally distributed, with $47 \%$ in a 'large' cell population and the remainder in a 'small' cell population. The median diameter of the large adipose cells was not associated with expression of inflammatory genes. Rather, the fraction of small adipose cells was consistently
\end{abstract}

\section{T. McLaughlin $(\varangle)$}

Division of Endocrinology,

Stanford University School of Medicine,

300 Pasteur Drive, Rm S025,

Stanford, CA 94305-5103, USA

e-mail: tmclaugh@stanford.edu

A. Deng $\cdot$ G. Yee $\cdot$ C. Lamendola $\cdot$ G. Reaven $\cdot$ P. S. Tsao

Department of Cardiovascular Medicine,

Stanford University School of Medicine,

Stanford, CA, USA

S. W. Cushman · A. Sherman

National Institute of Diabetes and Digestive and Kidney Diseases,

National Institutes of Health,

Bethesda, MD, USA associated with inflammatory gene expression, independently of sex, insulin resistance and BMI. This association was more pronounced in insulin-resistant than insulin-sensitive individuals. Insulin resistance also independently predicted expression of inflammatory genes.

Conclusions/interpretation This study demonstrates that among moderately obese, weight-stable individuals an increased proportion of small adipose cells is associated with inflammation in subcutaneous adipose tissue, whereas size of mature adipose cells is not. The observed association between small adipose cells and inflammation may reflect impaired adipogenesis and/or terminal differentiation. However, it is unclear whether this is a cause or consequence of inflammation. This question and whether small vs large adipose cells contribute differently to inflammation in adipose tissue are topics for future research.

\section{Trial registration: ClinicalTrials.gov NCT00285844}

Funding: National Institutes of Health/ National Institute of Diabetes and Digestive and Kidney Diseases 1 R01 DK071309-01, 5RO1DK071333, 5K23 RR16071, Clinical and Translational Science Award 1UL1 RR025744, and the National Institute of Diabetes and Digestive and Kidney Diseases Intramural Research Program.

Keywords Adipocyte - Adipose tissue - Inflammation · Insulin resistance $\cdot$ Obesity
Abbreviations
CLS Crown-like structures
$\mathrm{C}_{\mathrm{t}} \quad$ Cycle threshold
IST Insulin suppression test
SSPG Steady-state plasma glucose
SSPI Steady-state plasma insulin 


\section{Introduction}

Obesity is associated with insulin resistance, which is the precursor to type 2 diabetes and cardiovascular disease. The mechanistic link between excess body weight and insulin resistance is unclear. Furthermore, not all moderately obese individuals are insulin-resistant. Indeed, insulin sensitivity can vary sixfold in this population [1]. We have taken advantage of this observation to compare molecular and cellular characteristics of subcutaneous abdominal adipose tissue from equally obese individuals, who were classified as insulin-resistant or insulin-sensitive, by the modified insulin suppression test (IST).

It has been suggested that biological characteristics of adipose tissue, rather than mass, might lead to insulin resistance. Specifically, large adipose cells are thought to contribute to insulin resistance via increased lipolysis $[2,3]$ and, as shown more recently, by inciting an inflammatory cascade $[4,5]$. The literature shows that increasing BMI and larger mean adipose cell size in humans and rodents are associated with greater density of macrophages in adipose tissue [6] and that large adipose cells appear necrotic and are surrounded by macrophages [4]. These studies, however, are non-quantitative, were not done in a weight-stable state and the single study with humans [4] included very obese participants without clear evidence of weight stability and with biopsies from different body regions.

We have previously shown that simply measuring the mean diameter of adipose cells or estimating size by dividing total lipid by total number of cells are inadequate ways of describing the size of adipose cells because size distribution is not unimodal, i.e. cells primarily reside in either a large or a small cell fraction, with few in-between [7]. Taking this into consideration, we have been able to perform more complex analyses of the relationship between cell size characteristics and both molecular and clinical characteristics of human participants. Thus, using healthy participants matched for BMI but with different degrees of insulin sensitivity, we examined the commonly cited hypothesis that larger adipose cell size is associated with inflammation in subcutaneous adipose tissue.

\section{Methods}

Participants Participants included 49 moderately obese, otherwise healthy adults selected from a larger participant pool recruited via newspaper advertisements in the cities surrounding Stanford University. Participants were required to be 35 to 65 years of age, free of major organ disease and non-diabetic as defined by fasting plasma glucose concentration $<7.0 \mathrm{mmol} / \mathrm{l}$, and to have BMI of 26 to $36 \mathrm{~kg} / \mathrm{m}^{2}$ and stable body weight for 3 months. Participants were required not to be engaged in a weight loss programme or taking lipid-lowering medications, including fish oil $/ n-3$ fatty acids, steroid preparations or medications for weight loss. Participants with a history of eating disorder, bariatric surgery or liposuction were excluded, as were pregnant or lactating women. All participants underwent quantification of insulin-mediated glucose disposal via a modified IST (see below) and a subcutaneous periumbilical adipose tissue biopsy. The study was approved by the Stanford University Human Participants Committee and all participants gave written, informed consent.

Quantification of insulin-mediated glucose disposal and other clinical measurements Insulin-mediated glucose disposal was quantified by a modification [8] of the IST as originally described and validated $[9,10]$. Briefly, participants were infused for $240 \mathrm{~min}$ with octreotide $\left(0.27 \mu \mathrm{g} \mathrm{m}^{-2} \mathrm{~min}^{-1}\right.$; to suppress endogenous insulin secretion), insulin ( $25 \mathrm{mU} \mathrm{m}^{-2} \mathrm{~min}^{-1}$ ) and glucose $\left(240 \mathrm{mg} \mathrm{m}^{-2} \mathrm{~min}^{-1}\right)$. Blood was drawn at $10 \mathrm{~min}$ intervals from 210 to $240 \mathrm{~min}$ of the infusion to measure plasma glucose and insulin concentrations, and the mean of these four values was used as the steady-state plasma insulin (SSPI) and steady-state plasma glucose (SSPG) concentrations for each individual. As SSPI concentrations are similar in all participants during these tests, the SSPG concentration provides a direct measure of the ability of insulin to mediate disposal of an infused glucose load, and the higher the SSPG concentration, the more insulinresistant the individual. Participants whose SSPG results were in the top or bottom 40th percentile were included and defined as insulin-resistant or insulin-sensitive, respectively. Individuals in the middle 20th percentile were excluded, as they were not clearly insulin-resistant or insulin-sensitive. This procedure identifies participants who are relatively homogeneous with respect to degree of obesity and general health, but who differ substantially with respect to insulin sensitivity.

Other clinical and laboratory measurements After a $12 \mathrm{~h}$ overnight fast, plasma glucose, insulin and lipid/lipoprotein concentrations were measured as previously described $[9$, 11]. Other experimental measurements included weight, height, calculated BMI, waist circumference measured at end-expiration at the point midway between the iliac crest and lower costal margin, race/ethnicity and blood pressure (average of six readings taken after sitting for $5 \mathrm{~min}$ ).

Subcutaneous abdominal fat biopsy and determination of cell size distribution Subcutaneous abdominal fat biopsies were performed under sterile conditions by scalpel incision inferior to the umbilicus, as previously described [6]. Two samples of 20 to $30 \mathrm{mg}$ of tissue were immediately fixed in osmium tetroxide and incubated in a water bath at $37^{\circ} \mathrm{C}$ for 
$48 \mathrm{~h}$ as previously described [12], for cell size distribution analysis using a multisizer device (Multisizer III; Beckman Coulter, Miami, FL, USA) with a $400 \mu \mathrm{m}$ aperture. The effective cell size range using this aperture is 20 to $240 \mu \mathrm{m}$. The instrument was set to count 6,000 particles and the fixed-cell suspension was diluted so that coincident counting was less than $10 \%$. After collection of pulse sizes, the data were expressed as particle diameters and displayed as histograms of counts against diameter using linear bins and a linear scale for the $\mathrm{x}$-axis. All participants displayed a roughly bimodal distribution of cells with a 'hump' of large cells and a 'tail' of small cells, separated by a cell-count 'nadir' as described previously [7]. Quantitative analyses to describe the cell size distribution were done via mathematical modelling using non-linear least squares function nls, $\mathrm{R}$ 1.9 (http://www.r-project.org/), in which a single formula using seven cell size variables was able to describe the individual data points of each participant:

$$
\begin{aligned}
y= & h 1 \exp ([x-x 0 / w 1])+h 2 \exp (-[x-x 0] / w 2) \\
& +h p \exp (-[x-c p] 2 / w p)
\end{aligned}
$$

where $x$ is the cell diameter and $x 0$ is the smallest diameter; $h 1$ and $w 1$ are the height and width of the first exponential; $h 2$ and $w 2$ are the height and width of the second exponential; and $h p, c p$ and $w p$ are the height, centre and width of the Gaussian curve, respectively. In the curve formulated by this equation, the 'tail' of small cells is represented by the sum of two exponentials and the 'hump' of large cells is represented by a Gaussian curve (Fig. 1). Endpoints of interest included $c p$ (peak centre), which represents the median diameter of the large cells, and the fraction of small cells, which is defined as the per cent of cells below the nadir.

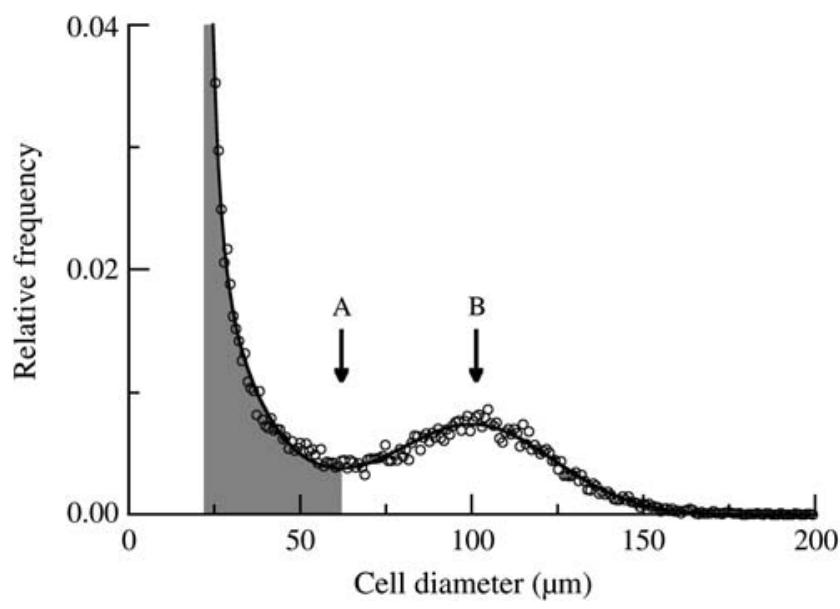

Fig. 1 Multisizer analysis of cell size distribution in a single patient, with large cells represented by a Gaussian formula and small cells represented by a double-exponential formula. Fraction of small cells (shaded) includes all cells below the nadir (arrow A). Peak centre is the cell diameter where the Gaussian hump peaks (arrow B)
Quantitative real-time PCR for markers of inflammation Total RNA was extracted from flash-frozen biopsy samples using TRIzol (Life Technologies, Gaithersburg, MD, USA) and kits (Adipose Tissue RNAeasy; Qiagen, Valencia, CA, USA) according to the manufacturers' instructions. After DNase treatment, cDNA was synthesised from $5 \mathrm{mg}$ of total RNA using Maloney murine leukaemia virus reverse transcriptase (SuperScript II kit; Invitrogen, Carlsbad, CA, USA). Taqman primer/probe sets for markers of inflammatory cell infiltrate (CD14 [also known as NDUFA2], CD45 [also known as PTPRC], CD68 and EMR-1) and inflammation $(I L-6, I L-8, i N O S, M C P-1$ [also known as CCL2] and $T N F-\alpha$ ), as well as for $18 \mathrm{~S}$ ribosomal RNA were obtained from Applied Biosystems (Foster City, CA, USA). Amplification was carried out in triplicate on an ABI Prism 7700 (Applied Biosystems) as follows: 2 min at $50^{\circ} \mathrm{C}$ and $10 \mathrm{~min}$ at $95^{\circ} \mathrm{C}$, followed by 40 cycles of $95^{\circ} \mathrm{C}$ for $15 \mathrm{~s}$ and $60^{\circ} \mathrm{C}$ for $1 \mathrm{~min}$. A threshold cycle $\left(\mathrm{C}_{\mathrm{t}}\right.$ value $)$ was obtained from each amplification curve and a $\Delta \mathrm{C}_{\mathrm{t}}$ value was first calculated by subtracting the $C_{t}$ value for $18 \mathrm{~S}$ ribosomal RNA from the $C_{t}$ value for each sample. $A \Delta \Delta C_{t}$ value was then calculated by subtracting the $\Delta \mathrm{C}_{\mathrm{t}}$ value of a single insulin-sensitive participant (control). Fold-changes compared with the control were then determined by raising 2 to the $\Delta \Delta \mathrm{C}_{\mathrm{t}}$ power.

Statistical analyses Data are presented as mean and standard deviation from the mean. Non-normally distributed variables (all inflammation genes except $M C P-1$ ) were log-transformed for statistical tests. Insulin sensitivity was treated as a categorical variable, with participants classified as being from the insulin-resistant or insulin-sensitive group. Multiple linear regression analysis with each inflammatory marker as the dependent variable was performed with the fraction of small cells, sex and insulin resistance group as predictors. Formal testing for interactions between fraction of small cells and both sex and insulin resistance group was done. Similar multiple regression models were constructed for prediction of each inflammatory gene with the peak diameter of the large cells as a predictor, along with sex and insulin resistance group. Again, formal testing for interactions between peak diameter and both sex and insulin resistance was done. A $p$ value of $p<0.05$ was considered statistically significant.

\section{Results}

Anthropometric and laboratory characteristics of 49 moderately obese individuals are shown in Table 1 . The range of BMI in this study was 25.3 to $34.8 \mathrm{~kg} / \mathrm{m}^{2}$. Approximately $60 \%$ of participants were women and $78 \%$ were 
Table 1 Demographic and clinical characteristics of study participants

\begin{tabular}{ll}
\hline Characteristic & Values (mean \pm SD or $n$ ) \\
\hline Participants $(n)$ & 49 \\
Age (years) & $52 \pm 9$ \\
Race $(n)$ & \\
White & 38 \\
Hispanic & 4 \\
Black & 2 \\
Asian & 5 \\
Sex (n) & \\
Women & 30 \\
Men & 19 \\
BMI (kg/m $\left.{ }^{2}\right)$ & $30.0 \pm 2.7$ \\
Weight (kg) & $88.6 \pm 15.0$ \\
Waist circumference (cm) & $102 \pm 11$ \\
Fasting blood glucose (mmol/l) & $5.4 \pm 0.4$ \\
Systolic BP (mmHg) & $125 \pm 14$ \\
Diastolic BP (mmHg) & $74 \pm 8$ \\
SSPG (mmol/l) & $8.7 \pm 3.2$ \\
Cholesterol (mmol/l) & $5.0 \pm 0.9$ \\
HDL-cholesterol (mmol/l) & $1.3 \pm 0.5$ \\
LDL-cholesterol (mmol/l) & $3.2 \pm 0.7$ \\
Triacylglycerol (mmol/l) & $1.4 \pm 0.8$ \\
\hline
\end{tabular}

white. Of the 49, 28 were insulin-resistant and 21 were insulin-sensitive, quantitated by the modified IST as described. Mean peak adipose cell diameter was $111 \pm$ $15 \mu \mathrm{m}$, mean fraction of small adipose cells was $53 \pm 10 \%$. Table 2 demonstrates the independent associations between fraction of small adipose cells and relative expression of eight inflammation genes in adipose tissue. Standardised correlation coefficients $(\beta)$ are shown for each predictor in the model (predictors were fraction of small cells, insulin resistance group and sex). Statistical adjustment for BMI did not alter results and it was removed from the final models.

It is clear from Table 2 that $C D 14, C D 45, E M R-1, I L-6$ and $M C P-1$ were associated with fractions of small cells, independently of insulin resistance group and sex. Interestingly, these markers include genes both non-specific (IL-6, $I L-8, M C P-1, E M R-1)$ and specific $(C D 14, C D 45)$ for monocyte lineage cells. Insulin resistance was also independently associated with these same genes, as well as with $C D 68$ and $I L-8$. Sex was not significantly associated with expression of any of the inflammation genes. Formal testing for interaction between insulin resistance group and fraction of small cells in prediction of inflammation genes revealed a statistically significant interaction only for EMR-1 ( $p<$ 0.02). A similar trend was noted for CD14 $(p=0.09)$.
Because insulin resistance group modified the relationship between fraction of small cells and inflammatory gene expression in some cases, correlation coefficients between inflammation genes and fraction of small adipose cells are shown separately for insulin-resistant and insulin-sensitive participants in Fig. 2. It is apparent that correlations were stronger for the insulin-resistant than for the insulin-

Table 2 Independent associations between fraction of small adipose cells and inflammatory genes, adjusted for insulin resistance group and sex

\begin{tabular}{|c|c|c|c|}
\hline Predictors per gene & $\beta \pm \mathrm{SE}$ & Standardised $\beta$ & $p$ value $^{\mathrm{a}}$ \\
\hline \multicolumn{4}{|l|}{$T N F-\alpha$} \\
\hline Fraction small cells & $0.002 \pm 0.10$ & -0.003 & 0.98 \\
\hline Insulin-resistant group & $0.03 \pm 0.02$ & 0.19 & 0.22 \\
\hline Sex & $0.03 \pm 0.02$ & 0.20 & 0.20 \\
\hline \multicolumn{4}{|l|}{$i N O S$} \\
\hline Fraction small cells & $-0.44 \pm 1.36$ & -0.05 & 0.74 \\
\hline Insulin-resistant group & $0.06 \pm 0.28$ & 0.03 & 0.84 \\
\hline Sex & $0.01 \pm 0.29$ & 0.008 & 0.96 \\
\hline \multicolumn{4}{|l|}{$C D 14$} \\
\hline Fraction small cells & $-1.62 \pm 0.63$ & 0.33 & 0.01 \\
\hline Insulin-resistant group & $0.35 \pm 0.13$ & 0.37 & 0.009 \\
\hline Sex & $0.07 \pm 0.13$ & 0.08 & .58 \\
\hline \multicolumn{4}{|l|}{$C D 45$} \\
\hline Fraction small cells & $2.29 \pm 0.87$ & 0.34 & 0.01 \\
\hline Insulin-resistant group & $0.46 \pm 0.18$ & 0.36 & 0.01 \\
\hline Sex & $-0.18 \pm 0.18$ & -0.14 & 0.31 \\
\hline \multicolumn{4}{|l|}{ CD68 } \\
\hline Fraction small cells & $0.21 \pm 0.64$ & 0.04 & 0.74 \\
\hline Insulin-resistant group & $0.53 \pm 0.73$ & 0.55 & 0.0002 \\
\hline Sex & $-0.08 \pm 0.14$ & -0.08 & 0.58 \\
\hline \multicolumn{4}{|l|}{ EMR-1 } \\
\hline Fraction small cells & $1.89 \pm 0.74$ & 0.33 & 0.01 \\
\hline Insulin-resistant group & $0.43 \pm 0.15$ & 0.39 & 0.007 \\
\hline Sex & $-0.14 \pm 0.16$ & -0.12 & 0.38 \\
\hline \multicolumn{4}{|l|}{ IL6 } \\
\hline Fraction small cells & $3.29 \pm 1.51$ & 0.28 & 0.04 \\
\hline Insulin-resistant group & $1.03 \pm 0.31$ & 0.45 & 0.002 \\
\hline Sex & $-0.05 \pm 0.32$ & -0.02 & 0.88 \\
\hline \multicolumn{4}{|l|}{ IL8 } \\
\hline Fraction small cells & $1.24 \pm 1.14$ & 0.13 & 0.28 \\
\hline Insulin-resistant group & $1.19 \pm 0.23$ & 0.63 & $<0.0001$ \\
\hline Sex & $-0.41 \pm 0.24$ & -0.22 & 0.09 \\
\hline \multicolumn{4}{|l|}{$M C P-1$} \\
\hline Fraction small cells & $0.20 \pm 0.06$ & 0.38 & 0.003 \\
\hline Insulin-resistant group & $0.04 \pm 0.01$ & 0.40 & 0.003 \\
\hline Sex & $0.003 \pm 0.01$ & 0.03 & 0.78 \\
\hline
\end{tabular}

${ }^{\mathrm{a}} p$ values are adjusted for sex and insulin resistance group via multiple linear regression analysis. Adjustment for BMI did not alter results 
Fig. 2 Relationship between fraction of small cells and inflammatory genes as shown in insulin-resistant $(\mathbf{a}-\mathbf{e})$ and insulin-sensitive ( $\mathbf{f}-\mathbf{j})$ men (white circles) and women (black circles)
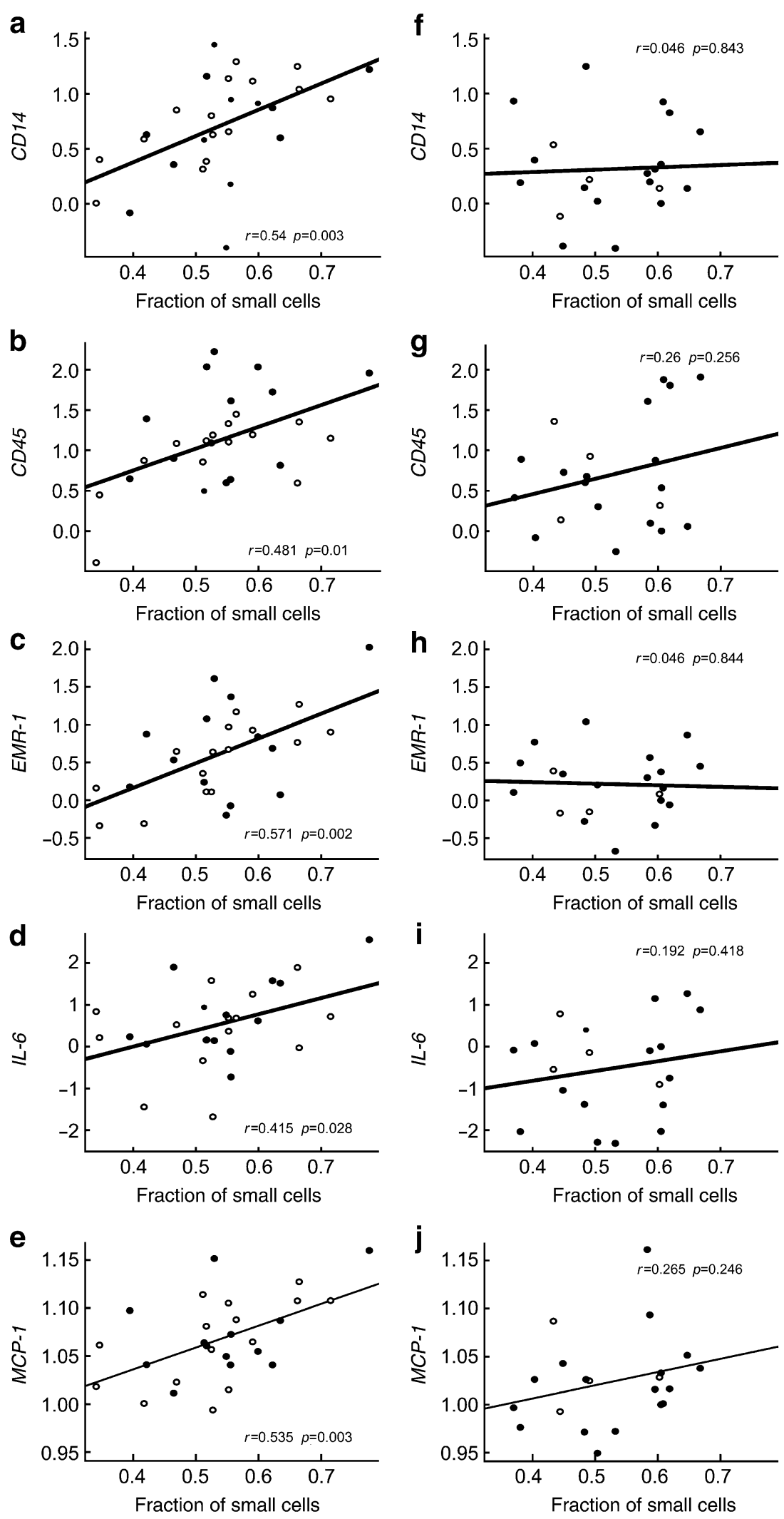
sensitive subgroup for all genes, most dramatically for CD14 and EMR-1.

Table 3 shows the independent relationships between peak centre (diameter of large adipose cells) and inflammatory gene expression. Peak centre was not significantly associated with any inflammatory genes. As before, insulin resistance independently predicted expression of seven $(C D 14, C D 45$, $C D 68, E M R-1, I L-6, I L-8, M C P-1)$ of the nine genes studied. Again, sex was not a significant predictor of inflammatory genes, although male sex bore an inverse association with $I L-8$ that was of borderline significance $(p=0.05)$.

\section{Discussion}

Our results demonstrate that among moderately obese healthy individuals, increased size of mature adipose cells is not associated with inflammation in subcutaneous adipose tissue. Rather, an increased proportion of small (vs large) adipose cells independently predicted expression of five inflammatory genes. This relationship was independent of insulin resistance, BMI and sex. These findings yield a new perspective on the purported relationship between adipose cell size characteristics and inflammation.
Table 3 Independent associations between peak centre of large adipose cells and inflammatory genes, adjusted for insulin resistance group and sex
${ }^{a} p$ values are adjusted for sex and insulin resistance group via multiple linear regression analysis. Adjustment for BMI did not alter results

\begin{tabular}{|c|c|c|c|}
\hline Predictors per gene & $\beta \pm \mathrm{SE}$ & Standardised $\beta$ & $p$ value $^{\mathrm{a}}$ \\
\hline \multicolumn{4}{|l|}{$T N F-\alpha$} \\
\hline Peak centre & $-0.006 \pm 0.0008$ & -0.11 & 0.49 \\
\hline Insulin-resistant group & $0.03 \pm 0.02$ & 0.22 & 0.16 \\
\hline Sex & $0.021 \pm 0.026$ & 0.14 & 0.44 \\
\hline \multicolumn{4}{|l|}{ iNOS } \\
\hline Peak centre & $-0.01 \pm 0.01$ & -0.20 & 0.26 \\
\hline Insulin-resistant group & $0.22 \pm 0.29$ & 0.08 & 0.61 \\
\hline Sex & $-0.16 \pm 0.33$ & -0.09 & 0.62 \\
\hline \multicolumn{4}{|l|}{$C D 14$} \\
\hline Peak centre & $0.007 \pm 0.007$ & 0.22 & 0.16 \\
\hline Insulin-resistant group & $0.256 \pm 0.14$ & 0.34 & 0.03 \\
\hline Sex & $0.24 \pm 0.16$ & 0.15 & 0.36 \\
\hline \multicolumn{4}{|l|}{$C D 45$} \\
\hline Peak centre & $0.004 \pm 0.007$ & 0.11 & 0.50 \\
\hline Insulin-resistant group & $0.47 \pm 0.19$ & 0.36 & 0.02 \\
\hline Sex & $-0.17 \pm 0.22$ & -0.13 & 0.45 \\
\hline \multicolumn{4}{|l|}{ CD68 } \\
\hline Peak centre & $0.0002 \pm 0.004$ & -0.08 & 0.60 \\
\hline Insulin-resistant group & $0.50 \pm 0.13$ & 0.58 & 0.0002 \\
\hline Sex & $-0.07 \pm 0.15$ & -0.15 & 0.34 \\
\hline \multicolumn{4}{|l|}{$E M R-1$} \\
\hline Peak centre & $0.001 \pm 0.006$ & 0.04 & 0.98 \\
\hline Insulin-resistant group & $0.46 \pm 0.17$ & 0.42 & 0.009 \\
\hline Sex & $-0.19 \pm 0.19$ & -0.16 & 0.34 \\
\hline \multicolumn{4}{|l|}{ IL6 } \\
\hline Peak centre & $-0.004 \pm 0.01$ & -0.06 & 0.70 \\
\hline Insulin-resistant group & $1.15 \pm 0.34$ & 0.50 & 0.002 \\
\hline Sex & $-0.21 \pm 0.38$ & -0.09 & 0.59 \\
\hline \multicolumn{4}{|l|}{ IL8 } \\
\hline Peak centre & $0.0007 \pm 0.008$ & -0.11 & 0.42 \\
\hline Insulin-resistant group & $1.27 \pm 0.25$ & 0.68 & $<0.0001$ \\
\hline Sex & $-0.55 \pm 0.28$ & -0.29 & 0.05 \\
\hline \multicolumn{4}{|l|}{$M C P-1$} \\
\hline Peak centre & $0.00004 \pm 0.0005$ & -0.04 & 0.80 \\
\hline Insulin-resistant group & $0.04 \pm 0.01$ & 0.45 & 0.004 \\
\hline Sex & $-0.004 \pm 0.017$ & -0.03 & 0.83 \\
\hline
\end{tabular}


These results are important because a number of studies in humans and mice have suggested that increased adipose cell diameter is associated with increased inflammation in adipose tissue [4, 6]. Evaluation of genetically obese mice, including those deficient in hormone-sensitive lipase, which develop large adipose cells in the absence of obesity, demonstrated increased density of macrophages localised to sites of single, apparently necrotic adipose cells that were termed 'crown-like structures' (CLS) and were increased up to 30 -fold [4]. In 12 obese (BMI $30-45 \mathrm{~kg} / \mathrm{m}^{2}$ ) humans with biopsies from gluteal and abdominal sites, mean adipocyte size also correlated with electron microscopically defined features of adipocyte death and CLS [4]. Larger adipose cell size has also been reported to predict insulin resistance and type 2 diabetes, independently of body fat [13]. These findings have led to speculation that enlargement of adipose cells triggers inflammation, which in turn contributes to insulin resistance [5]. Our results do not confirm this hypothesis.

Discrepancies between our results and those of others [4, 6] may be due to the fact that most published data are on overfed mice. These mice, which had multiple CLS surrounding apparently necrotic, large adipocytes, had mean adipocyte diameters that were multiple-fold increased in comparison to the controls [4]. In our moderately obese human participants, adipose cell diameters were only about $40 \%$ greater than that described in lean individuals [14]; moreover, all participants were weight-stable. Limited data in humans showed inflammation and CLS in association with large adipose cells primarily in extremely obese (BMI $30-45 \mathrm{~kg} / \mathrm{m}^{2}$ ) humans [6]. Our BMI range was somewhat restricted, thereby limiting the ability to see an association between adipose cell size (reflecting degree of obesity) and inflammation. Furthermore, we excluded morbidly obese participants and thus our findings do not rule out the possibility of such an association in the higher BMI range.

The apparent contradiction between our results and those of prior studies $[4,6]$ may also derive from the use of the multisizer methodology, which allowed us to look beyond [1] cell size as estimated from a small portion of tissue visualised microscopically or [2] mean cell size estimated from total lipid extracted and total cell count. Neither of these methods are as quantitative and accurate as the multisizer method, with the former sampling only a small portion of the total adipose tissue and the latter potentially generating erroneous conclusions, if cell size is distributed in a bimodal fashion, as we have previously shown [7]. Our results were adjusted statistically for insulin sensitivity, sex and BMI, confirming that the lack of association between peak cell diameter and inflammation was free of confounding.

Turning to the second major finding of this study, there was a consistent positive association between increased fraction of small adipose cells and inflammation in adipose tissue. This association was independent of insulin resistance, which was also independently associated with inflammation. Insulin resistance status significantly modified the relationship between small cell fraction and inflammation for $E M R-1$, with the relationship being stronger in the insulin-resistant subgroup. A similar trend was noted for CD14 $(p=0.09)$ and several other inflammatory genes (NS). These findings should be interpreted in the context of previous data showing that inflammatory markers and macrophage density are higher in insulinresistant than in equally obese insulin-sensitive individuals [15]. Our ascertainment that the relationship between inflammation and small adipose cells was independent of insulin resistance is therefore important as such. Indeed, it appears that an increased proportion of small adipose cells is associated with inflammation in subcutaneous adipose tissue.

The significance of the increased proportion of small cells is not clear. In our previous analysis showing that the insulin-resistant subgroup of moderately obese participants is characterised by an higher proportion of small adipose cells as compared with insulin-sensitive, BMI-matched controls [7], gene expression of differentiation markers was also decreased in the insulin-resistant group, leading us to conclude that the small cells represented immature cells with impaired ability to store triacylglycerol. A number of other human studies also provide support for the notion that impaired adipogenesis characterises the insulin-resistant state, in that they show decreased expression of differentiation and/or fat storage genes in individuals with type 2 diabetes or insulin resistance as compared with controls [16-19]. Thus, one interpretation of the current results is that impaired differentiation of adipocytes is associated with inflammation in subcutaneous adipose tissue, which in turn is related to insulin resistance.

In-vitro studies support a relationship between inflammation and impaired adipocyte differentiation/ fat storage. For example, incubation of 3T3-L1 pre-adipocytes with IL6 or TNF- $\alpha$ impairs terminal differentiation and results in decreased triacylglycerol storage. Pre-adipocytes accumulate little lipid in the setting of IL- 6 and almost no lipid in the setting of TNF- $\alpha$, maintaining a fibroblast-like appearance and exhibiting a higher migration rate that leads to cluster formations, consistent with a proliferative state [20]. In our study, differentiation genes were decreased and inflammatory cytokines increased, suggesting that inflammation in adipose tissue may incite a cascade leading not only to decreased terminal differentiation, but also to increased inflammatory activity of adipocytes. This notion is supported by the demonstration that when 3T3-L1 adipocytes are co-cultured with monocytes, secretion of TNF- $\alpha$ from the monocytes stimulates elaboration of IL-6, IL-8, monocyte chemotactic protein-1 (MCP-1) and TNF- $\alpha$ 
from the adipocytes, a process that is prevented in the presence of TNF- $\alpha$-blocking antibody [21]. Thus, inflammation external to adipose cells may impair their differentiation and fat storage capacity, and may even incite further inflammation.

An alternative explanation for the association between small adipose cells and inflammation in subcutaneous adipose tissue is that the small cells act in a proinflammatory manner. In this context it is important to note that the small cells are adipose cells and not macrophages, as evidenced by prior investigations, in which we isolated adipose cells by flotation and continued to observe the bimodal distribution by multisizer analysis [7]. Furthermore, the aperture range for multisizer analysis excludes cells with a diameter of less than $20 \mu \mathrm{m}$, essentially excluding cells of monocyte lineage, while electron microscopy demonstrates that even the small cells are smooth and round, whereas contrasting ruggatedappearing macrophages adhere to the surfaces of the larger cells. While our results do not make clear whether inflammation in adipose tissue is a product of monocyte/ macrophages or adipocytokines, the current data do show an association between increased fraction of small adipose cells and inflammatory markers that are non-specific and specific for monocyte-lineage cells. These findings complement and extend a prior study, in which we found upregulation of inflammatory genes, both specific and non-specific for monocyte-lineage cells, in association with insulin resistance [15], although histological sections showed relatively sparse macrophages in insulin-resistant and insulin-sensitive participants. On the other hand, Weisberg et al [6] showed that inflammation in adipose tissue could be traced to cells that were of bone marrow origin and thus of monocyte lineage. In all, it is most likely that at least part of the inflammation in subcutaneous adipose tissue is attributable to adipose cells themselves. At this point, however, it is unclear whether large or small adipose cells function differently in this respect. Further research is needed to clarify this issue.

Finally, it is important to consider how the observed relationships in the current study relate to insulin resistance. In this regard, our findings corroborate and extend our prior finding, in 29 women, that inflammation in subcutaneous adipose tissue is associated with insulin resistance independently of obesity [15]. The current study includes men and again demonstrates a consistent relationship between insulin resistance and inflammation, which is independent of BMI and also of sex. Importantly, our prior study did not include cell size data, whereas the current study, by adjusting for potential confounding by adipose cell size or fraction of small cells, demonstrates that the relationship between insulin resistance and inflammation is independent of differences in cell size variables. We have now demonstrated a relationship between increased proportion of small cells and inflammation, both of which are significantly associated with insulin resistance $[7,15]$. Thus a triad is suggested, in which increased proportion of small cells, inflammation in subcutaneous adipose tissue and systemic insulin resistance are associated.

This study has several limitations. First, it would have been instructive to compare inflammatory markers in small vs large adipose cells, but separation of cells by size is difficult and requires larger amounts of tissue than we were able to obtain in this study. To our knowledge, there are no published studies addressing this topic. Second, we did not measure genes related to differentiation/fat storage in this analysis. In a prior study [7], however, we showed that insulin-resistant participants had decreased expression of differentiation markers, as well as an increased proportion of small adipose cells, indicating that impairment of adipose cell differentiation characterises the insulinresistant state. Finally, our study population was primarily white, limiting its generalisability to other races. Importantly, we were able to assess women and men, demonstrating no independent sex effect on inflammation or on the relationship between cell size variables and inflammation.

In conclusion, inflammation in subcutaneous adipose tissue of moderately obese, weight-stable individuals appears not to be associated with increased size of mature adipose cells, but rather with a relative predominance of cells in the 'small cell' phase of development. Considered in the context of prior findings that inflammation is associated with insulin resistance and that a predominance of small cells is also associated with insulin resistance, the following hypotheses about the biological properties of adipose tissue that contribute to the development of insulin resistance are possible and are specifically supported by our results: (1) inflammation impairs differentiation and/or maturation of pre-adipocytes into mature adipose cells; (2) individuals with impaired adipose cell differentiation develop inflammation; and (3) the small adipose cells demonstrate biological activity similar to inflammatory cells. Future work addressing these possibilities, including studies separating large and small adipose cells, will shed further light on these emerging concepts of the metabolic and morphological mechanisms by which subcutaneous adipose tissue can promote insulin resistance independently of total body mass.

Duality of interest The authors declare that there is no duality of interest associated with this manuscript.

\section{References}

1. McLaughlin T, Abbasi F, Lamendola C, Reaven G (2006) Heterogeneity in prevalence of risk factors for cardiovascular disease and type 2 diabetes in obese individuals: impact of differences in insulin sensitivity. Arch Intern Med 167:642-648 
2. Jacobsson B, Smith U (1972) Effect of cell size on lipolysis and antilipolytic action of insulin in human fat cells. J Lipid Res 13:651-656

3. Ostman J, Backman L, Hallberg D (1975) Cell size and the antilipolytic effect of insulin in human subcutaneous adipose tissue. Diabetologia 11:159-164

4. Cinti S, Mitchell G, Barbatelli G et al (2005) Adipocyte death defines macrophage localization and function in adipose tissue of obese mice and humans. J Lipid Res 46:2347-2355

5. Weller KE, Hotamisligil GS (2003) Obesity-induced inflammatory changes in adipose tissue. J Clin Invest 112:1785-1788

6. Weisberg S, McCann D, Desai M, Rosenbaum M, Leibel R, Ferrante A (2003) Obesity is associated with macrophage accumulation in adipose tissue. J Clin Invest 112:1796-1808

7. McLaughlin T, Sherman A, Tsao P et al (2007) Enhanced proportion of small adipose cells in insulin-resistant vs insulinsensitive obese individuals implicates impaired adipogenesis. Diabetologia 50:1707-1715

8. Pei D, Jones CNO, Bhargava R, Chen Y-DI, Reaven GM (1994) Evaluation of octreotide to assess insulin-mediated glucose disposal by the insulin suppression test. Diabetologia 37:843-845

9. Shen S-W, Reaven GM, Farquhar JW (1970) Comparison of impedance to insulin mediated glucose uptake in normal and diabetic subjects. J Clin Invest 49:2151-2160

10. Greenfield MS, Doberne L, Kraemer FB, Tobey TA, Reaven GM (1981) Assessment of insulin resistance with the insulin suppression test and the euglycemic clamp. Diabetes 30:387-392

11. Carantoni M, Abbasi F, Chu L et al (1997) Adherence of mononuclear cells to endothelium in vitro is increased in patients with NIDDM. Diabetes Care 20:1462-1465

12. Stern JS, Batchelor BR, Hollander N, Cohn CK, Hirsch J (1972) Adipose cell size and immunoreactive insulin levels in obese and normal weight adults. Lancet ii:948-951
13. Weyer C, Foley JE, Bogardus C, Tataranni PA, Pratley RE (2000) Enlarged subcutaneous abdominal adipocyte size, but not obesity itself, predicts type II diabetes independent of insulin resistance. Diabetologia 43:1498-1506

14. Krotkiewski M, Bjorntorp P, Sjostrom L, Smith U (1983) Impact of obesity on metabolism in men and women: importance of regional adipose tissue distribution. J Clin Invest 72:1150-1162

15. McLaughlin T, Deng A, Gonzales O et al (2008) Insulin resistance is associated with a modest increase in inflammation in subcutaneous adipose tissue of moderately obese women. Diabetologia 51:2303-2308

16. Yang X, Jansson PA, Nagaev I et al (2004) Evidence of impaired adipogenesis in insulin resistance. BBRC 317:1045-1051

17. Dubois S, Heilbronn LK, Smith SR, Albu JB, Kelley ER (2006) Decreased expression of adipogenic genes in obese subjects with type 2 diabetes. Obesity 14:1543-1552

18. Jan V, Cervera P, Maachi M et al (2004) Altered fat differentiation and adipocytokine expression are inter-related and linked to morphological changes and insulin resistance in HIV-1 infected lipodystrophic patients. Antivir Ther 9:555-564

19. Bastard JP, Caron M, Vidal H et al (2002) Association between altered expression of adipogenic factor SREBP1 in lipoatrophic adipose tissue from HIV-1-infected patients and abnormal adipocyte differentiation and insulin resistance. Lancet 359: 1026-1031

20. Gustafson B, Smith U (2006) Cytokines promote WNT signaling and inflammation and impair the normal differentiation and lipid accumulation in 3T3-L1 preadipocytes. J Biol Chem 281:95079516

21. Suganami T, Nishida J, Ogawa Y (2005) A paracrine loop between adipocytes and macrophages aggravates inflammatory changes. Arterioscler Thromb Vasc Biol 25:2062-2068 